\title{
Protective Effect of Basic Fibroblast Growth Factor Against Myocyte Death and Arrhythmias in Acute Myocardial Infarction in Rats
}

\author{
Satoru Nishida, MD; Hiroshi Nagamine, MD; Yoko Tanaka, Go Watanabe, MD
}

\begin{abstract}
The present study in rats investigated whether basic fibroblast growth factor (bFGF) plays an important role in cardioprotection against myocardial cell death and arrhythmias in acute myocardial infarction (AMI). After ligating the left coronary artery in 62 Wistar rats, $20 \mu \mathrm{g}$ of human recombinant bFGF was injected into the infarcted myocardium in 33 rats (group F), while saline was used for 29 control rats (group C). The development of ventricular tachyarrhythmias was assessed during the first $30 \mathrm{~min}$ of ischemia. After $24 \mathrm{~h}$ occlusion, the hearts of the surviving rats (group $F: n=13$, group $C: n=10$ ) were excised to assess minimum infarct wall thickness and infarct size, determine the number of TUNEL-positive cardiomyocytes and to analyze Bcl-2 and Bax expression by immunohistochemical staining and Western blotting. The incidence of ventricular tachycardia was higher in group $C$ than in group $F(p<0.05)$. The thinning ratio was higher in group $F$ than in group $C(p<0.05)$. There were fewer TUNEL-positive cardiomyocytes in the infarct border area in group $\mathrm{F}$ than in group $\mathrm{C}(\mathrm{p}<.0001)$. Western blot analysis showed greater expression of Bcl-2 in group $\mathrm{F}$ than in group $\mathrm{C}(\mathrm{p}<0.05)$, but similar expression of Bax in the 2 groups. In conclusion, intramyocardial administration of bFGF prevented ischemia-induced myocardial cell death and arrhythmias. (Circ J 2003; 67: 334-339)
\end{abstract}

Key Words: Apoptosis; Arrhythmia; Basic fibroblast growth factor; Bcl-2

B asic fibroblast growth factor (bFGF) is a single-chain peptide with a molecular weight of $17 \mathrm{kD}$ and is referred to as heparin-binding growth factor 2 because of its affinity for heparin! bFGF induces endothelial and smooth muscle cell proliferation in vitro and angiogenesis in vivo, including the migration and proliferation of endothelial cells, vascular tube formation, and linkage to preexisting vascular networks $1,2 \mathrm{bFGF}$ has been detected in the infarcted myocardial tissue of the $\operatorname{dog}^{3,4}$ and in recent years, the use of bFGF to improve blood flow and increase the vascular number in the presence of tissue ischemia has been proposed, based on the results from animal experiments 5,6 In a canine myocardial infarct model, intracoronary injection of exogenous bFGF increased the number of arterioles and capillaries in the infarcted tissue, reduced infarct size, and improved cardiac systolic function; ${ }^{4}$ however, the mechanism of action of bFGF in the setting of acute myocardial infarction (AMI) is unclear?,8

Irreversible damage occurs in acutely ischemic myocardium within 4 to $6 \mathrm{~h}^{9}$ and true angiogenesis can only improve blood flow over the course of days? Kajstura et al ${ }^{10}$ and Fliss et $\mathrm{a}^{11}$ have recently demonstrated that in the rat heart induces apoptosis in the ischemic myocardial cells and that this might cause late myocyte death. Necrosis and apoptosis are 2 types of cardiac cell death associated with myocardial ischemia and whether cardiac cells die of apoptosis or necrosis is critical for the development of

(Received March 20, 2002; revised manuscript received January 20 , 2003; accepted February 4, 2003)

Department of Surgery (I), Kanazawa University School of Medicine, Kanazawa, Japan

Mailing address: Satoru Nishida, MD, Department of Surgery (I), Kanazawa University School of Medicine, Takaramachi 13-1, Kanazawa 920-8641, Japan. E-mail: snishida@k6.dion.ne.jp therapeutic strategies, because apoptosis is a process that may be altered by pharmacologic interventions. bFGF has been shown to be an important survival antiapoptotic factor in a variety of cell types! $!^{2,13}$ Cuevas et $\mathrm{al}^{14}$ described the antiapoptotic effect of acidic fibroblast growth factor $(\mathrm{aFGF})$ in a rat model of transient myocardial ischemia, ${ }^{14}$ but the antiapoptotic effects of bFGF in cardiomyocytes has not been investigated previously. We hypothesized that myocardial salvage associated with the administration of bFGF may be mediated by the prevention of myocardial apoptosis.

On the other hand, it has been shown that bFGF is localized in cardiac gap junctions 15 which suggests a role for bFGF in the control of cardiac rhythm. The purpose of the present study was to determine the effects of intramyocardial administration of bFGF on cardiomyocyte apoptosis and cardiac arrhythmias in a rat model of AMI.

\section{Methods}

\section{Animal Preparation}

Sixty-two adult male (270-300 g) Wistar rats were anesthetized by the intraperitoneal administration of $30 \mathrm{mg} / \mathrm{kg}$ sodium pentobarbital and were mechanically ventilated using a volume respirator (Model SN-480-7, Shinano, Tokyo, Japan). The electrocardiogram was obtained with platinum needle electrodes inserted subcutaneously. The animals received humane care as outlined in the 'The Guide for the Care and Use of Laboratory Animals' published by the USA National Institutes of Health (NIH Publication No. 85-23, revised 1996). A left lateral thoracotomy was performed through the fourth intercostal space and AMI was induced by ligating the proximal portion of the left coronary artery. Sham-operated rats were subjected to the 
identical treatment without the tying of the coronary ligature. After the coronary ligation, the rats were divided into 2 groups: Group $F(n=33)$ received human recombinant

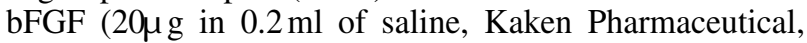
Tokyo, Japan) into the ischemic myocardium using a $27 \mathrm{~g}$ needle, while group $\mathrm{C}$ (control group, $n=29$ ) received the same volume of saline in the same manner. After these procedures, the chest was closed.

\section{Arrhythmia Assessment and Survival Rate}

Ischemia-induced ventricular arrhythmias were analyzed during the first $30 \mathrm{~min}$ of ischemia according to the Lambeth Convention guidelines for the analysis of experimental arrhythmias. ${ }^{6}$ The incidence of ventricular tachycardias and ventricular fibrillation were noted. Ventricular tachycardia was defined as a run of 6 or more beats at a rate faster than resting sinus rhythm, and ventricular fibrillation was defined as a signal for which the individual QRS complex could no longer be identified.

The survival rate was recorded after $24 \mathrm{~h}$ occlusion. The surviving rats were anesthetized and mechanically ventilated in the same manner. After they were killed by an intravenous injection of sodium pentobarbital, the heart was excised.

\section{Assessment of Infarct Size}

The heart was transected parallel to the atrioventricular groove at the center of the infarct area. The distal portion of the heart was fixed with $10 \%$ formalin for subsequent hematoxylin-eosin (H\&E) staining, terminal deoxynucleotidyl transferase-mediated dUTP-biotin nick end labeling (TUNEL) and immunohistochemical staining for Bcl-2 and Bax. The proximal portion was cut into 2-mm thick slices that were incubated in 1\% 2, 3, 5-triphenyl tetrazolium chloride (TTC; Sigma Chemical, St Louis, MO, USA) in phosphate buffered saline (PBS) for $10 \mathrm{~min}$ at room temperature, followed by fixation in $10 \%$ formalin for $60 \mathrm{~min}$. The slices were then digitally scanned (GT-9600; EPSON, Tokyo, Japan) and the images were analyzed using the National Institute of Health (NIH) image program. Infarct area was identified as an area unstained by TTC and infarct size was expressed as the ratio of infarct area to the whole left ventricular area. The rest of the proximal portion of the ventricle was frozen for subsequent Western blotting of Bcl-2 and Bax (Fig 1A).

\section{Left Ventricular Morphologic Analysis}

The sections of H\&E stained tissue were used to analyze left ventricular morphology. The degree of thinning of the infarcted wall was assessed by the thinning ratio, which was defined as the ratio of the minimum infarct wall thickness to normal septal wall thickness.

\section{Detection of TUNEL-Positive Cardiomyocytes}

Using paraffin sections that were $4 \mu \mathrm{m}$ thick, TUNEL was performed using the Apoptosis in situ Detection Kit (Wako, Osaka, Japan). Deparaffinized sections were washed with distilled water and treated with protein digestion enzyme for $5 \mathrm{~min}$ at $37^{\circ} \mathrm{C}$. After washing with 3 changes of $0.01 \mathrm{~mol} / \mathrm{L} \mathrm{PBS}$, sections were treated with terminal deoxynucleotidyl transferase (TdT) solution, incubated with $3 \%$ hydrogen peroxide for 5 min to block endogenous peroxidase activity, and then treated with peroxidase-conjugated antibody for $10 \mathrm{~min}$ at room temperature. After washing with $0.01 \mathrm{~mol} / \mathrm{L}$ PBS, nick end-labeling was visualized
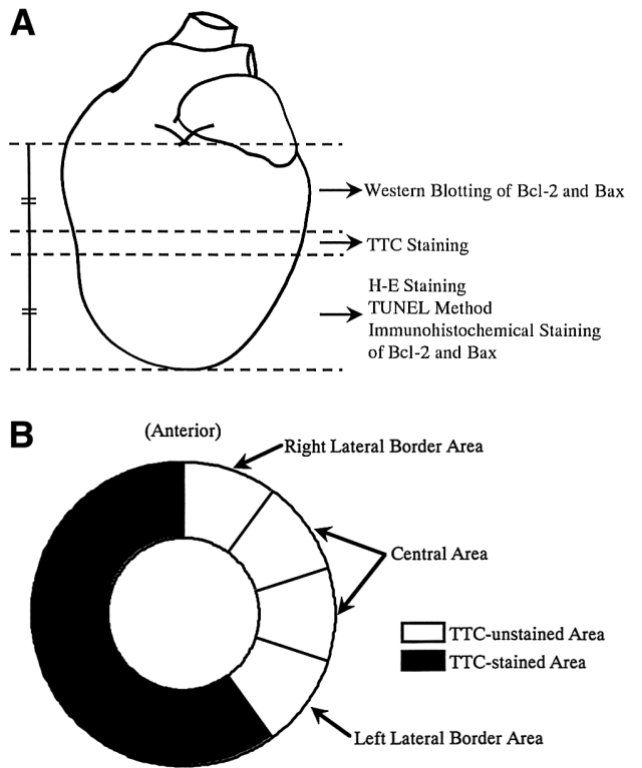

Fig 1. Heart slices and myocardial segments for assessing the distribution of TUNEL-positive cardiomyocytes. Three slices of the left ventricle were used to measure the infarcted area, for H\&E staining, for TUNEL staining, for immunohistochemical staining of $\mathrm{Bcl}-2$ and Bax, and for western blotting of Bcl-2 and Bax (A). The infarcted area was divided into 4 radial segments (right lateral border area, 2 central segments, and left lateral border area). Measurement of TUNEL-positive cardiomyocytes was performed in each segment (B).

by immersing the sections in 3,3'-diaminobenzidine solution with $0.006 \%$ hydrogen peroxide and counterstaining with hematoxylin. As a negative control, tissue sections were incubated with TdT buffer that did not contain the enzyme. As a positive control, tissue sections were treated with DNase I prior to treatment with TdT.

To assess the distribution of TUNEL-positive cardiomyocytes in the left ventricular wall, the infarcted area was divided into 4 radial segments, which included the right lateral border area adjacent to the interventricular septum, 2 central segments, and the left lateral border area adjacent to the left ventricular posterior wall. For each of the segments, color video images of 5 separate fields were captured randomly and digitized using an $\times 40$ objective on an Olympus Provis microscope. We determined the ratio between cardiomyocytes with TdT-stained nuclei (TUNEL-positive cardiomyocytes) and the total number of cardiomyocytes within the infarct area in the 5 fields. TUNEL-positive cardiomyocytes were carefully distinguished from non-cardiomyocytes, such as macrophages.

\section{Immunohistochemical Staining of Bcl-2 and Bax}

The immunohistochemical staining for Bcl-2 and Bax used paraffin sections that were $4 \mu \mathrm{m}$ thick. After the sections were deparaffinized, epitope unmasking was achieved by one of 2 methods.

Method 1 For immunohistochemical staining of Bcl-2, sections were incubated in $0.5 \mathrm{mg} / \mathrm{ml}$ of protease $(\mathrm{p}-4798$, Sigma) for $30 \mathrm{~min}$ at room temperature 17

Method 2 For immunohistochemical staining of Bax, sections were treated 3 times with microwave irradiation $(500 \mathrm{~W})$ for $5 \mathrm{~min}$ in $0.1 \mathrm{~mol} / \mathrm{L}$ citrate buffer $(\mathrm{pH} 6.0)$.

Intrinsic peroxidase activity was inhibited by the addition of $3 \%$ hydrogen peroxide, and nonspecific binding 
A
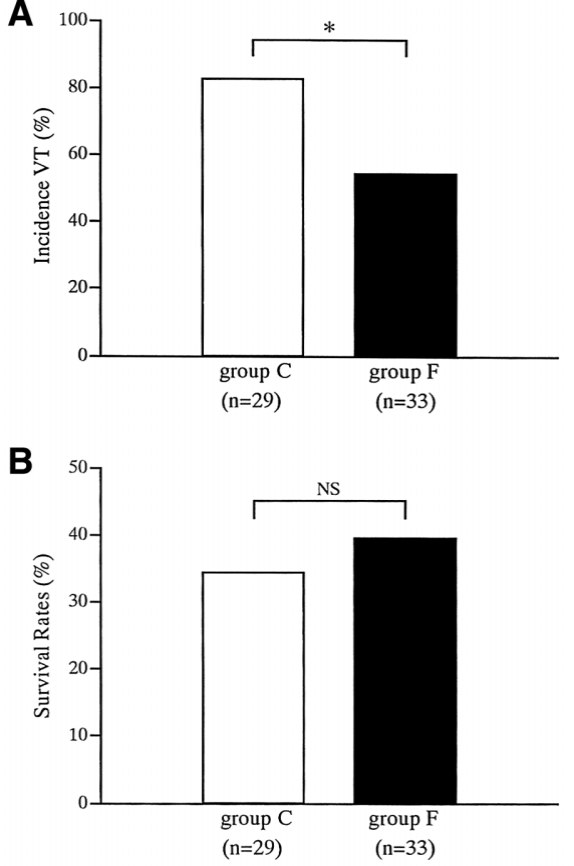

Fig 2. Incidence of ventricular tachycardia (VT; A) and survival rates $(B)$ in rats subjected to coronary artery occlusion. Group $\mathrm{C}$, infarcted rats $(n=29)$ treated with saline; group $F$, infarcted rats $(n=33)$ treated with bFGF $(20 \mu \mathrm{g}) . * \mathrm{p}<0.05$ vs control.

was blocked with normal goat serum. Rabbit polyclonal antibodies against either rat Bcl-2 ( $\triangle \mathrm{C} 21$, Santa Cruz, CA, USA) or rat Bax (P-19, Santa Cruz) were used as the primary antibodies, which were diluted 1:50 and incubated with the tissue sections for $45 \mathrm{~min}$ at room temperature. The secondary antibody, an anti-mouse/anti-rabbit labeled polymer, prepared by combining amino acid polymers with peroxidase and goat anti-mouse immunoglobulin (Ig) and anti-rabbit Ig reduced to Fab' fragments (Nichirei, Japan), was incubated with the tissue sections for $30 \mathrm{~min}$ at room temperature. Sections were then stained with $0.2 \mathrm{mg} / \mathrm{ml}$ of 3,3'-diaminobenzidine tetrahydrochloride for $10 \mathrm{~min}$ at room temperature. Between each step, the sections were washed with distilled water or $10 \mathrm{mmol} / \mathrm{L}$ sodium PBS (pH 7.2). The sections were then counterstained with hematoxylin.

\section{Western Blot Analysis of Bcl-2 and Bax}

Total protein was extracted from normal and infarcted areas of the left ventricle. Approximately $60 \mathrm{mg}$ of heart tissue was placed in $300 \mu 1$ lysis buffer (1\% Nonidet P-40, $1 \%$ sodium deoxycholate: $0.1 \%$ sodium dodecyl sulfate [SDS] in $1 \times \mathrm{PBS}$ ), and homogenized at $4^{\circ} \mathrm{C}$ for $20 \mathrm{~s}$, incubated on ice for $30 \mathrm{~min}$, centrifuged at $15,000 \mathrm{G}$ for $10 \mathrm{~min}$, and the supernatant removed. Protein concentration was measured using a BioRad model 550 microplate reader. Total protein $(50 \mu \mathrm{g})$ was mixed with loading buffer (B7709S, New England BioLabs, MA, USA), boiled for $3 \mathrm{~min}$, and loaded onto a 4-12\% Bis-Tris gel (NP0322 NuPAGE, Invitrogen, San Diego, CA, USA). Gels were run with a full range molecular weight ladder (Rainbow MW marker, Amersham Pharmacia, Biotech UK Ltd, Bucks, UK) and a Jurkat cell lysate (sc-2204, Santa Cruz) as a positive control sample. Proteins were transferred to an Immobilon-P membrane (IPVH 00010, Millipore, MA) by semidry blotting. Mem-
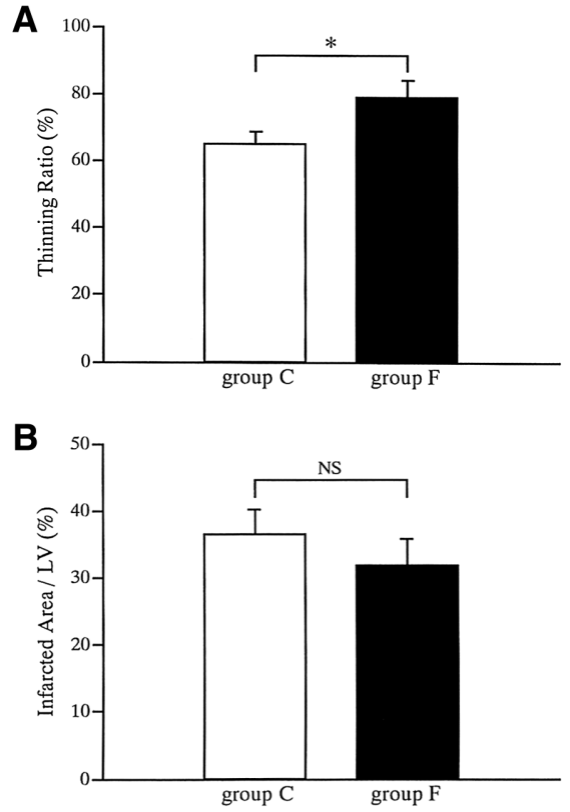

Fig 3. Effect of bFGF treatment on the thinning ratio (A) and infarct size (B). ${ }^{*} \mathrm{p}<0.05$ vs control.

branes were blocked with $5 \%$ milk in $1 \times$ PBS-Tween ( $20 \mathrm{mmol} / \mathrm{L}$ Tris $\mathrm{HCl} \mathrm{pH} 7.6,137 \mathrm{mmol} / \mathrm{L} \mathrm{NaCl}, 0.05 \%$ Tween-20) for $1 \mathrm{~h}$ at room temperature. Membranes were subsequently exposed to rabbit polyclonal anti-rat Bcl-2 $(\triangle \mathrm{C} 21$, Santa Cruz) or rabbit polyclonal anti-rat Bax (P-19, Santa Cruz) at 1:500 dilution in PBS-Tween for $3 \mathrm{~h}$. Bound antibody was detected by horseradish peroxidase conjugated anti-rabbit IgG. Finally, enhanced chemiluminescence (ECL) detection reagents were employed to visualize the peroxidase reaction products (Amersham). Bcl-2 was detected as a 26-kDa band and Bax as a 21-kDa band. The intensities of the bands were quantified using the NIH image program. Band densities were normalized to a sham operation control sample run on all gels.

\section{Statistical Analysis}

Data are expressed as the mean \pm standard error of the mean (SEM), with the exception of the percent incidence of arrhythmic events and survival rate, which were analyzed by chi-square analysis. The thinning ratio, infarct size, and Bcl-2 and Bax expressions were analyzed using an unpaired $t$ test. Analyses of TUNEL-positive cardiomyocytes from different myocardial regions were performed using two-way ANOVA followed by Fisher's post hoc comparison. A value of $\mathrm{p}<0.05$ was considered statistically significant.

\section{Results}

Arrhythmia Assessment and Survival Rate

The incidence of ventricular tachycardia was significantly higher in group $\mathrm{C}$ than in group $\mathrm{F}(82.8 \%$ vs $54.5 \%$, respectively, $\mathrm{p}<0.05$, Fig $2 \mathrm{~A}$ ), but the incidence of ventricular fibrillation was similar in the 2 groups $(41.1 \%$ in group $\mathrm{C}$ and $33.3 \%$ in group $\mathrm{F}$ ). The survival rate was $34.5 \%$ (10 of 29 rats) in group $\mathrm{C}$ and $39.4 \%$ (13 of 33 rats) in group $\mathrm{F}$ (NS, Fig 2B). 


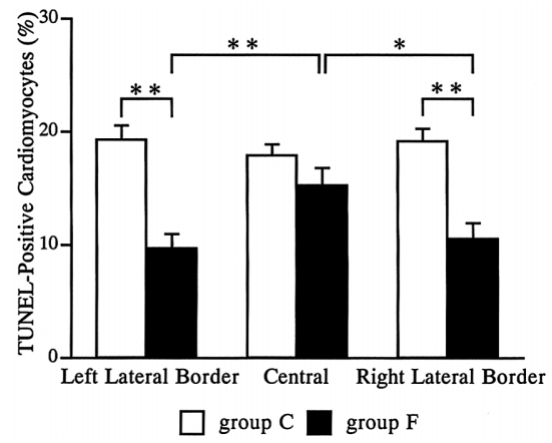

Fig 4. TUNEL-positive cardiomyocytes in myocardial segments within the infarcted area. ${ }^{*} \mathrm{p}<0.01,{ }^{*} \mathrm{p}<0.05$.

\section{Left Ventricular Morphologic Analysis and Infarct Size}

The left ventricular thinning ratio was significantly higher in group $\mathrm{F}$ than in group $\mathrm{C}(79.3 \pm 4.9 \%$ in group $\mathrm{F}$ $[n=13]$ and $64.5 \pm 4.1 \%$ in group $C[n=10], p<0.05$, Fig $3 A)$. The infarct size, which is expressed as the ratio of infarct area to total left ventricular (LV) area, tended to be smaller in group $\mathrm{F}$, although the difference was not statistically significant $(36.6 \pm 3.6 \%$ in group $C$ and $31.8 \pm 4.0 \%$ in group F, Fig 3B).

\section{TUNEL-Positive Cardiomyocytes}

In group $\mathrm{C}$, the percentage of TUNEL-positive cardiomyocytes was similar in all areas, but in group F, the percentage of TUNEL-positive cardiomyocytes was significantly lower in the left and right lateral border areas compared with the central segment ( $p<0.05$ for both). The percentage of TUNEL-positive cardiomyocytes was significantly reduced in the left and right lateral border areas in group F compared with group C (Fig 4).

\section{Immunohistochemical Localization and Western Blot} Analysis of Bcl-2 and Bax

Bcl-2-positive cardiomyocytes were observed diffusely in the LV free wall (Fig 5A), whereas Bax-positive cardio-
A

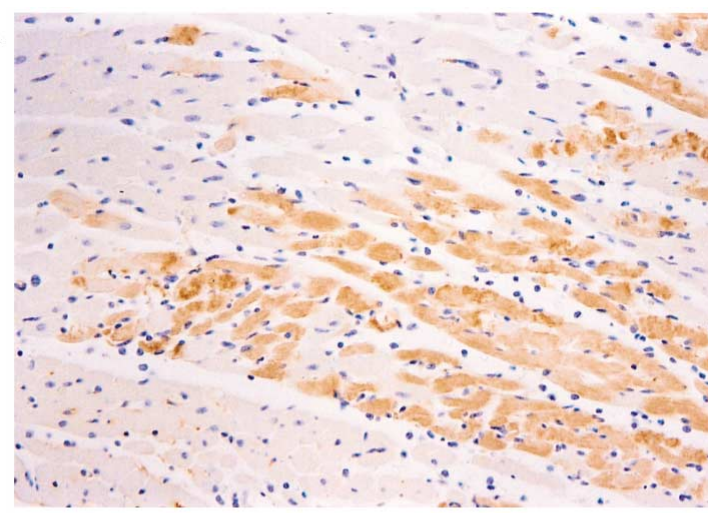

B

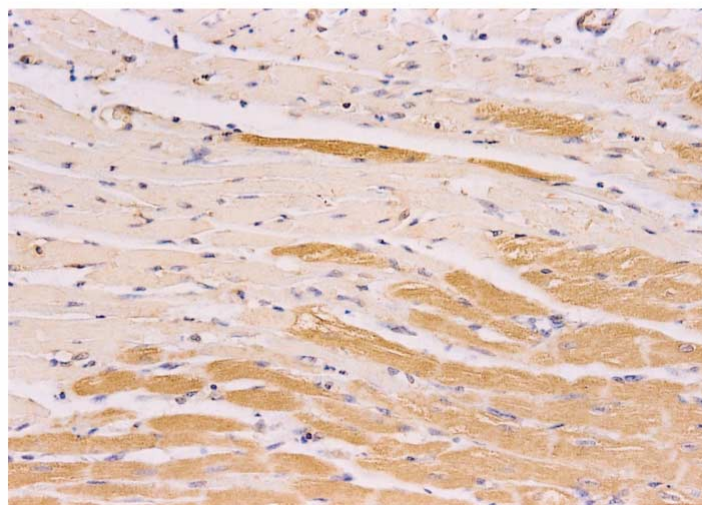

Fig 5. Immunohistochemical localization of Bcl-2 (A) and Bax (B) in group $\mathrm{C}$ showed that the distribution of Bcl-2- and Bax-positive cardiomyocytes was similar in the 2 groups.

myocytes were distributed throughout the left ventricle (Fig 5B). The distribution of Bcl-2 and Bax-positive cardiomyocytes was similar in the 2 groups. Western blot analysis for $\mathrm{Bcl}-2$ and Bax expression in the LV myocardium are shown in Fig 6A. Normalized densities for the Bcl-2 signal were significantly greater in group $\mathrm{F}$ than in group $\mathrm{C}$

\section{A} Bcl-2

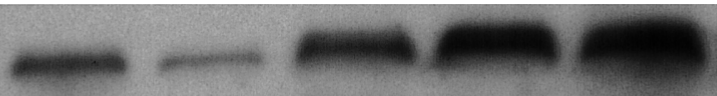

$\operatorname{Bax}$

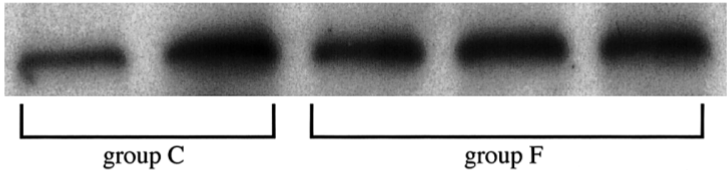

B

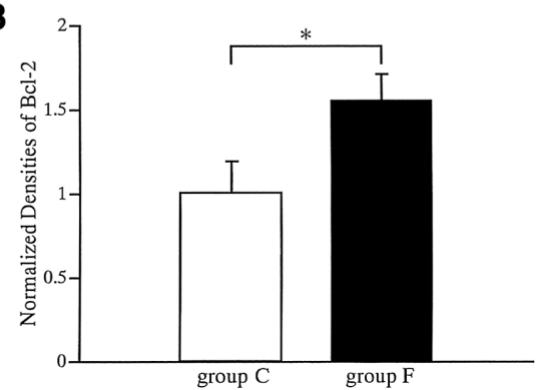

C

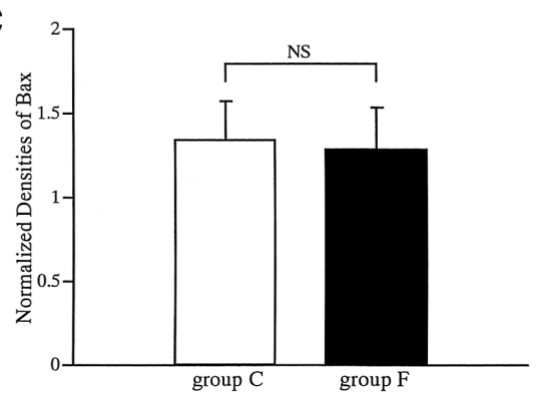

Fig 6. Western blot analysis of Bcl-2 and Bax in left ventricular myocardium (A). Normalized densities for Bcl-2 (B). Normalized densities for $\operatorname{Bax}(\mathrm{C}) .{ }^{*} \mathrm{p}<0.05$. 
$(1.01 \pm 0.18$ in group $\mathrm{C}$ vs $1.55 \pm 0.16$ in group $\mathrm{F}, \mathrm{p}<0.05$, Fig 6B), although the normalized densities for the Bax signal were similar in the 2 groups (Fig 6C).

\section{Discussion}

We recently reported that intramyocardial administration of bFGF increased regional myocardial blood flow and capillary and arteriolar density, reduced thinning of the infarcted region, and improved ventricular function in a canine myocardial infarct model ${ }^{18}$ however, in the infarct and border zone the administration of bFGF increased myocardial blood flow at 3 days and up to 1 week after AMI, respectively, which raises questions concerning the mechanism of action of bFGF in the setting of acute infarction. Specifically, irreversible damage occurs in acutely ischemic myocardium within 4 to $6 \mathrm{~h}$ of acute infarction, and true angiogenesis can only improve blood flow over the course of days.

The present study demonstrates that the intramyocardial administration of bFGF reduced the number of TUNELpositive cardiomyocytes in the infarct border area and upregulated the expression of $\mathrm{Bcl}-2$ in rats that underwent $24 \mathrm{~h}$ coronary occlusion. These results show that bFGF may prevent myocardial apoptosis triggered by acute ischemia. TUNEL detects single-strand DNA breaks as well as double-strand DNA breaks with resulting free 3'-OH termini. Therefore, TUNEL may not be specific for apoptosis, and some necrotic cells may be TUNEL-positive. However, it provides valuable information concerning the tissue distribution and cell types undergoing cell death. To evaluate the effect of bFGF on apoptosis, we performed semiquantitative assessment of $\mathrm{Bcl}-2$ and Bax expression, which are important modulators of apoptosis because Bcl-2 promotes cell survival and Bax accelerates cell death. The present study demonstrates that $\mathrm{Bcl}-2$ expression is significantly greater in bFGF-treated hearts than in control hearts. The ability of bFGF to promote Bcl-2 upregulation may explain, at least in part, its beneficial effects in the infarcted myocardium. The results in our study provide the first evidence that administration of bFGF counteracts cardiomyocyte apoptosis associated with coronary occlusion.

Kondo et al have demonstrated that removing bFGF or administering anti-bFGF monoclonal antibody induces apoptosis in murine aortic endothelial cells, and that constitutive expression of $\mathrm{Bcl}-2$ using gene transfer techniques decreases apoptosis induced by removing $\mathrm{bFGF}^{19}$ which suggests a potential bFGF-Bcl-2 interaction. Karsan et al have demonstrated that $\mathrm{bFGF}$ inhibits endothelial apoptosis by upregulating $\mathrm{Bcl}-2$, but not Mcl-1, Bcl-XL, or Bax? Bcl-2 expression was not upregulated at early time points, but bFGF had a protective effect without Bcl-2 upregulation. Therefore, bFGF inhibits endothelial cell apoptosis by Bcl-2-dependent and independent mechanism. In our study, the administration of bFGF caused overexpression of Bcl2 , but not Bax and we hypothesize that the cardioprotective effect of bFGF is related to Bcl-2 upregulation.

With respect to myocardial remodeling, it has been reported that in the rat heart there is very early remodeling of the myocardial infarct, a process that is characterized by extensive dilation of the left ventricular cavity and marked thinning of the infarcted left ventricular wall? ${ }^{21}$ In our study, the minimum wall thickness of the infarcted tissue was maintained by the administration of bFGF, suggesting that bFGF is effective in inhibiting early remodeling. This find- ing could be partially attributed to the attenuation of cardiomyocyte apoptosis; we previously reported that intramyocardial administration of bFGF reduces left ventricular remodeling with an increase in regional myocardial perfusion and angiogenesis 4 weeks after coronary occlusion in a canine model 18 Therefore, administration of bFGF preventing left ventricular remodeling by preventing myocardial apoptosis before angiogenesis can occur.

The infarct size, determined by TTC staining, was also reduced by the administration of bFGF, although the difference was not statistically significant. The capacity of bFGF to limit infarct size following intracoronary injection was initially reported by Yanagisawa-Miwa et al in $\operatorname{dogs}^{4}$ and we are currently investigating the effect of bFGF on infarct size reduction in a canine model. Compared with dogs, rats have few native collaterals 22 and it is likely that the effect of infarct size reduction is determined by the collateral blood supply and the animal species. In our study, the infarct size was determined as the ratio of infarct area to the whole left ventricular area, but if we examined the infarct size as the ratio of infarct area to the area at risk, it might be significantly reduced.

Our results show a reduction in the incidence of ischemia-induced ventricular tachycardia in bFGF-treated rats. Cuevas et al demonstrated that in a rat model of myocardial ischemia and reperfusion, the administration of aFGF just before coronary reperfusion reduced the incidence of ventricular tachycardia and ventricular fibrillation, ${ }^{23}$ but the anti-arrhythmic effect of bFGF has not been investigated in previous studies.

Although the present study shows an anti-arrhythmic effect of bFGF, the molecular mechanisms underlying this effect require further investigation. It has been reported that bFGF localizes to cardiac gap junctions, ${ }^{15}$ which is potentially a very significant observation because it suggests that bFGF exerts a regulatory influence on the intercellular communication of such junctions and therefore may regulate the coordination of contractility and cardiac rhythm. These findings suggest that the anti-arrhythmic effects of bFGF may be mediated through the cardiac gap junction channels and we hypothesize that the administration of bFGF could be a beneficial adjunctive component of antiarrhythmic therapy in the setting of AMI.

In conclusion, intramyocardial administration of bFGF in the setting of myocardial ischemia is cardioprotective, preventing the myocardial apoptosis and ventricular tachyarrhythmias triggered by acute myocardial ischemia. Intramyocardial administration of bFGF may be a new therapeutic approach for patients with AMI.

\section{Acknowledgment}

The authors thank Kaken Pharmaceutical Co Ltd for providing human recombinant $b F G F$.

\section{References}

1. Folkman J, Klagsbrun M. Angiogenic factors. Science 1987; 235: $442-447$.

2. Montesano R, Vassalli JD, Baird A, Guillemin R, Orci L. Basic fibroblast growth factor induces angiogenesis in vitro. Proc Natl Acad Sci USA 1986; 83: 7297-7301.

3. Kardami E, Fandrich RR. Basic fibroblast growth factor in atria and ventricles of the vertebrate heart. J Cell Biol 1989; 109: 1865-1875.

4. Yanagisawa-Miwa A, Uchida Y, Nakamura F, Tomaru T, Kido H, Kamijo T, et al. Salvage of infarcted myocardium by angiogenic action of basic fibroblast growth factor. Science 1992; 257: 1401 1403. 
5. Yamamoto T, Suto N, Okubo T, Mikuniya A, Hanada H, Yagihashi $\mathrm{S}$, et al. Intramyocardial delivery of basic fibroblast growth factorimpregnated gelatin hydrogel microspheres enhances collateral circulation to infarcted canine myocardium. Jpn Circ J 2001; 65: 439444.

6. Iwatate M, Miura T, Ikeda Y, Kawamura S, Dairaku Y, Okamura T, et al. Effects of in vivo gene transfer of fibroblast growth factor-2 on cardiac function and collateral vessel formation in the microembolized rabbit heart. Jpn Circ J 2001; 65: 226-231.

7. Unger EF, Banai S, Shou M, Lazarous DF, Jaklitsch MT, Scheinowitz $\mathrm{M}$, et al. Basic fibroblast growth factor enhances myocardial collateral flow in a canine model. Am J Physiol 1994; 266: H1588-H1595.

8. Horrigan MC, MacIsaac AI, Nicolini FA, Vince DG, Lee P, Ellis SG, et al. Reduction in myocardial infarct size by basic fibroblast growth factor after temporary coronary occlusion in a canine model. Circulation 1996; 94: 1927-1933.

9. DeWood MA, Spores J, Notske R, Mouser LT, Burroughs R, Golden MS, et al. Prevalence of total coronary occlusion during the early hours of transmural myocardial infarction. $N$ Engl J Med 1980; 303: $897-802$.

10. Kajstura J, Cheng W, Reiss K, Clark WA, Sonnenblick EH, Krajewski S, et al. Apoptotic and necrotic myocyte cell deaths are independent contributing variables of infarct size in rats. Lab Invest 1996; 74: 86-107.

11. Fliss H, Gattinger D. Apoptosis in ischemic and reperfused rat myocardium. Circ Res 1996; 79: 949-956.

12. Desire L, Courtois Y, Jeanny JC. Endogenous and exogenous fibroblast growth factor 2 support survival of chick retinal neurons by control of neuronal neuronal bcl-x(L) and bcl-2 expression through a fibroblast berowth factor receptor 1- and ERK-dependent pathway. $J$ Neurochem 2000; 75: 151-163.

13. Wang Y, He H, Zigler JS Jr, Iwata T, Ibaraki N, Reddy VN, et al. bFGF suppresses serum-deprivation-induced apoptosis in a human lens epithelial cell line. Exp Cell Res 1999; 249: 123-130.

14. Cuevas P, Reimers D, Carceller F, Martinez-Coso V, RedondoHorcajo M, Saenz de Tejada I, et al. Fibroblast growth factor-prevents myocardial apoptosis triggered by ischemia reperfusion injury.
Eur J Med Res 1997; 2: 465-468.

15. Kardami E, Stoski RM, Doble BW, Yamamoto T, Hertzberg EL, Nagy JI. Biochemical and ultrastructural evidence for the association of basic fibroblast growth factor with cardiac gap junctions. J Biol Chem 1991; 266: $19551-19557$.

16. Walker MJ, Curtis MJ, Hearse DJ, Campbell RW, Janse MJ, Yellon $\mathrm{DM}$, et al. The Lambeth Conventions: Guidelines for the study of arrhythmias in ischaemia infarction, and reperfusion. Cardiovasc Res 1988; 22: 447-455.

17. Finley JC, Grossman GH, Dimeo P, Petrusz P. Somatostatin-containing neurons in the rat brain: Widespread distribution revealed by immunocytochemistry after pretreatment with pronase. Am J Anat 1978; 153: 483-488.

18. Kawasuji M, Nagamine H, Ikeda M, Sakakibara N, Takemura H, Fujii S, et al. Therapeutic angiogenesis with intramyocardial administration of basic fibroblast growth factor. Ann Thorac Surg 2000; 69: $1155-1161$.

19. Kondo S, Yin D, Aoki T, Takahashi JA, Morimura T, Takeuchi J. bcl-gene prevents apoptosis of basic fibroblast growth factordeprived murine aortic endothelial cells. Exp Cell Res 1994; 213: $428-432$.

20. Karsan A, Yee E, Poirier GG, Zhou P, Craig R, Harlan JM. Fibroblast growth factor-2 inhibits endothelial cell apoptosis by Bcl-2dependent and independent mechanisms. Am J Pathol 1997; 151: $1775-1784$.

21. Hearse DJ, Richard V, Yellon DM, Kingma JG Jr. Evolving myocardial infarction in the rat in vivo: An inappropriate model for the investigation of drug-induced infarct size limitation during sustained regional ischaemia. J Cardiovasc Pharmacol 1988; 11: 701-710.

22. Maxwell MP, Hearse DJ, Yellon DM. Species variation in the coronary collateral circulation during regional myocardial ischaemia: A critical determinant of the rate of evolution and extent of myocardial infarction. Cardiovasc Res 1987; 21: 737-746.

23. Cuevas P, Carceller F, Hernandez-Madrid A, Cuevas B, MartinezCoso V, Gimenez-Gallego G. Protective effects of acidic fibroblast growth factor against cardiac arrhythmias induced by ischemia and reperfusion in rats. Eur J Med Res 1997; 2: 33-36. 